\title{
Influence of spacecraft outgassing on the exploration of tenuous atmospheres with in situ mass spectrometry
}

\author{
B. Schläppi, ${ }^{1}$ K. Altwegg, ${ }^{1}$ H. Balsiger, ${ }^{1}$ M. Hässig, ${ }^{1}$ A. Jäckel, ${ }^{1}$ P. Wurz, ${ }^{1}$ B. Fiethe, ${ }^{2}$ \\ M. Rubin, ${ }^{3}$ S. A. Fuselier, ${ }^{4}$ J. J. Berthelier, ${ }^{5}$ J. De Keyser, ${ }^{6}$ H. Rème, ${ }^{7,8}$ and U. Mall ${ }^{9}$ \\ Received 1 June 2010; revised 5 August 2010; accepted 24 August 2010; published 14 December 2010.
}

[1] In situ mass spectrometry has been a powerful tool in many space missions to investigate atmospheres and exospheres of different bodies in the solar system. Applying new technologies, the mass spectrometers have become increasingly more sensitive. In this study, we show that spacecraft outgassing, which can never be completely prevented, will be the limiting factor in future missions that investigate very tenuous atmospheres and exospheres of moons, asteroids, or comets at large heliocentric distances. The Rosetta Orbiter Spectrometer for Ion and Neutral Analysis (ROSINA) instrument on the European Space Agency Rosetta mission has monitored spacecraft outgassing for 6 years during \#9the cruise phase with unprecedented instrument sensitivity. It is shown that diffusion of gas from materials and from the spacecraft interior plays an important role in maintaining a relatively permanent thin gas cloud around the spacecraft for many years. The density \#9and composition of this gas cloud depends on location on the spacecraft, maneuvers, and payload activity. The main contaminants are water, which is adsorbed on cold surfaces, and organics from the spacecraft structure, electronics, and insulations. Decomposed lubricant material can give a significant contribution to the total background. Fortunately for Rosetta, outgassing of the spacecraft will play a minor role when the comet is close to perihelion; only in the early phase of the mission the outgassing may be larger than the cometary signature.

Citation: Schläppi, B., et al. (2010), Influence of spacecraft outgassing on the exploration of tenuous atmospheres with in situ mass spectrometry, J. Geophys. Res., 115, A12313, doi:10.1029/2010JA015734.

\section{Introduction}

[2] The contamination of a spacecraft in space by its own outgassing has been a point of concern for some time. It is well known that critical optical surfaces including mirrors, windows, thermal control surfaces, and solar cells are susceptible to contaminants such as organic materials and water. Therefore, materials used in spacecraft are tested and carefully selected for their outgassing behavior. Contamination control is a key issue in building scientific spacecraft. Nevertheless, it is well known from different spacecraft that, after some time in space, optical surfaces acquire a layer of contaminants that can be significant and that can degrade

\footnotetext{
${ }^{1}$ Physikalisches Institut, University of Bern, Bern, Switzerland.

${ }^{2}$ Institute of Computer and Network Engineering, TU Braunschweig, Braunschweig, Germany.

${ }^{3}$ AOSS, University of Michigan, Ann Arbor, Michigan, USA.

${ }^{4}$ Space Physics Department, Lockheed Martin Advanced Technology Center, Palo Alto, California, USA.

${ }^{5}$ LATMOS, Saint-Maur, France.

${ }^{6}$ Space Physics Division, BIRA-IASB, Brussels, Belgium.

${ }^{7}$ UPS, CESR, University of Toulouse, Toulouse, France.

${ }^{8}$ CNRS, UMR 5187, Toulouse, France.

${ }^{9}$ Max-Planck-Institut für Sonnensystemforschung, Katlenburg-Lindau, Germany.

Copyright 2010 by the American Geophysical Union. 0148-0227/10/2010JA015734
}

instrument performance. For example, a gradual deterioration in performance has been reported for the Solar Extreme UV Monitor (SEM) of the Charge, Element, and Isotope Analysis System CELIAS on the Solar and Heliospheric Observatory (SOHO), a joint NASA/European Space Agency (ESA) project. This performance degradation is consistent with an exponential deposition of $15 \mathrm{~nm}$ of carbon over 1300 days [McMullin et al., 2002], which corresponds to a permanent mean particle density of organics around the spacecraft of $\sim 5 \cdot 10^{4} \mathrm{~cm}^{-3}$. This deposition is nonvolatile. For the same spacecraft, the deposition of an ice layer was determined from deterioration of the EUV imaging telescope performance. The thickness of this layer reaches up to $70 \AA$ A per month [Defise et al., 1997]. In 5 years, a deposition up to $420 \mathrm{~nm}$ is possible. Assuming water is dominant, this corresponds to a mean permanent water molecule density of $\sim 5 \cdot 10^{5} \mathrm{~cm}^{-3}$ or a partial pressure of $\sim 10^{-11}$ mbar (with an assumed temperature of $150 \mathrm{~K}$ ). This ice layer can be removed by heating the camera or by turning the surface with the deposition into the Sun.

[3] Several missions (e.g., Stardust [Bhaskaran et al., 2004], Cassini-Huygens [Haemmerle and Gerhard, 2006], Chandra X-Ray Observatory [Plucinsky et al., 2003], and others) experienced unexpected deterioration of their camera systems. All of these missions underwent very rigorous contamination control programs during the fabrication, integration, and test up 
to launch. In most cases, contamination could be removed in flight by carefully heating the subsystems, sometimes over very long times. An extensive database of different contamination measurements is given by Green [2001].

[4] Outgassing leads to different problems for scientific in situ mass spectrometers than those experienced by optical systems: although instrument performance is not affected, as such, a limit of detectability for atmospheric neutral and charged particles is given by the gaseous spacecraft environment. For example, the lunar orbital mass spectrometer experiment in the Apollo 15 orbital science package suffered severely from spacecraft outgassing despite being mounted on a boom $7.3 \mathrm{~m}$ away from the spacecraft main body [Hoffman et al., 1972]. The impact of contamination by the spacecraft environment is particularly important for any mission trying to analyze thin atmospheres of comets, asteroids, or moons, which are at large heliocentric distance or have little volatile material.

[5] Thus far, the gaseous environment of a spacecraft has been monitored mostly by using quartz crystal monitors that accumulate organic material preferentially [e.g., Wood et al., 1997; Brinza et al., 2000]. The only long-term observation of the gaseous and particle environment of a spacecraft, where the density and the composition of the neutral gas and the particle impacts have been monitored, is the Midcourse Space Experiment (MSX) [Uy et al., 2003]. The spacecraft was launched in 1996 into an orbit of $900 \mathrm{~km}$ from the Earth. This spacecraft was carefully designed with respect to cleanliness considerations because it carried sensitive optical instruments. However, the total pressure sensor as well as the neutral mass spectrometer on MSX reported significant outgassing even after 7 years in space. In particular, water, which initially decreased, was still present well above its expected level. Water vapor measurements showed pressure peaks during spacecraft slews and payload activity. The authors suggested that water is easily adsorbed in multilayer insulation (MLI) and that this insulation represents a large reservoir for outgassing of water.

[6] Cassini-Huygens also has a mass spectrometer for thermal neutral and ionized gas, the Ion and Neutral Mass Spectrometer (INMS) [Waite et al., 2004]. No measurable spacecraft outgassing was reported by INMS although the camera system on the same spacecraft suffered severely from deposits because of outgassing [Haemmerle and Gerhard, 2006]. The limit of detection of the INMS at $2 \cdot 10^{5} \mathrm{~cm}^{-3}$ [Waite et al., 2004] or about $5 \cdot 10^{-12} \mathrm{mbar}$ (with a temperature of $150 \mathrm{~K}$ ) for this open ionization source sets an upper limit on Cassini spacecraft outgassing. In contrast to the camera, where contamination was detected quite early in the mission, the INMS acquired mass spectra only on arrival at Titan, after about 7 years of interplanetary cruise.

[7] We report here results from the cruise phase of the ESA Rosetta mission to comet $67 \mathrm{P} / \mathrm{Churyumov-Gerasimenko}$ [Glassmeier et al., 2007]. Rosetta was launched in March 2004 and will encounter its target comet in 2014. To match the orbit of the comet, three Earth flybys and a Mars flyby were performed. The minimum heliocentric distance was reached in 2004 at $0.883 \mathrm{AU}$; the farthest distance thus far was in early 2009 at 2.2 AU. Rosetta encountered the asteroid Steins in September 2008 at a flyby distance of $800 \mathrm{~km}$, had its third Earth flyby in November 2009, and is currently on its way to its $5 \mathrm{AU}$ aphelion. It will approach the comet in 2014 at a heliocentric distance of about 4 AU.
At this distance, outgassing from the comet is expected to be weak [Schulz et al., 2004], and therefore outgassing of the spacecraft may well be the limiting factor in detecting cometary material.

[8] Rosetta has two mass spectrometers, ROSINA-Double Focusing Mass Spectrometer (DFMS) and ROSINAReflectron-type Time of Flight (RTOF) mass spectrometer, and a pressure sensor, ROSINA-Comet Pressure Sensor (COPS) [Balsiger et al., 2007], onboard, which measure neutral and ionized volatile material in the cometary coma. Both mass spectrometers have unprecedented sensitivity, which allows measurement of very low neutral particle densities. The Rosetta spacecraft will remain in close proximity to the comet for an extended period of time, and the relative velocity of the cometary coma and spacecraft will be close to zero. Under these conditions, the two populations, outgassing material from the spacecraft and cometary atmosphere and ionosphere, cannot be discriminated by their energy. It is therefore very important to know spacecraft backgrounds before arriving at the comet.

[9] In the present study, we discuss outgassing of the spacecraft as a function of time since launch, heliocentric distance, spacecraft maneuvers, and payload activity.

\section{Spacecraft and Instruments}

[10] A sketch of the spacecraft is given in Figure 1. Rosetta is approximately a cube with sides of $2.0 \mathrm{~m} \times$ $2.1 \mathrm{~m} \times 2.8 \mathrm{~m}$ and has $64 \mathrm{~m}^{2}(2 \mathrm{~m} \times 16 \mathrm{~m}$ on each side of the spacecraft) solar cells mounted on booms extending from the cube. Rosetta was designed to be a very clean spacecraft, not only because of its mass spectrometers, but also because of its optical instruments (e.g., UV spectrometer and optical camera). Outgassing requirements were very stringent for the spacecraft as well as for the payload. MLI is completely sealed on all panels, except the $-z$ panel, where the spacecraft is vented. The spacecraft has thrusters on all eight corners and on the $-\mathrm{z}$ panel. All thrusters are shielded from direct view of the payload, which is located mostly on the $+z$ panel. Normal attitude control is done with reaction wheels. Thrusters are used to offload the wheels approximately once per week. On the $+y$ and $-y$ panels, there are louvers to control temperature. The high gain antenna is attached to the $+x$ panel and is made of a carbon fiber sandwich structure [Pereira, 2001]. The lander (PHILAE) is attached to the $-\mathrm{x}$ panel, which also contains cold surfaces for cooling the infrared spectrometer VIRTIS [Coradini et al., 2007]. The lander panel is normally kept out of direct sunlight. The solar aspect angle (SAA), defined as the angle between spacecraft-Sun vector and $\mathrm{S} / \mathrm{C}+\mathrm{z}$ axis, is almost always within $\pm 5^{\circ}$ of the normal to the solar cells. In the $x-z$ plane, the SAA varies normally between $+15^{\circ}$ and $+175^{\circ}$ from the $+z$ axis. Far from the Sun, the angle can be larger than $180^{\circ}$ for a limited period, thus illuminating the $-x$ (lander) panel. The positions of the two mass spectrometers and of the pressure sensor COPS are also shown in Figure 1. In this study, we present data from all three ROSINA sensors. The main field of view (FOV) $\left(20^{\circ} \times 20^{\circ}\right)$ of DFMS is in the $+z$ direction. In addition, DFMS has an opening with a field of view $\left(5^{\circ} \times 5^{\circ}\right)$ in the $+x$ direction. The field of view of RTOF is $10^{\circ} \times 40^{\circ}$ in the direction of $+z$. The fields of view of both mass spectrometers exclude 


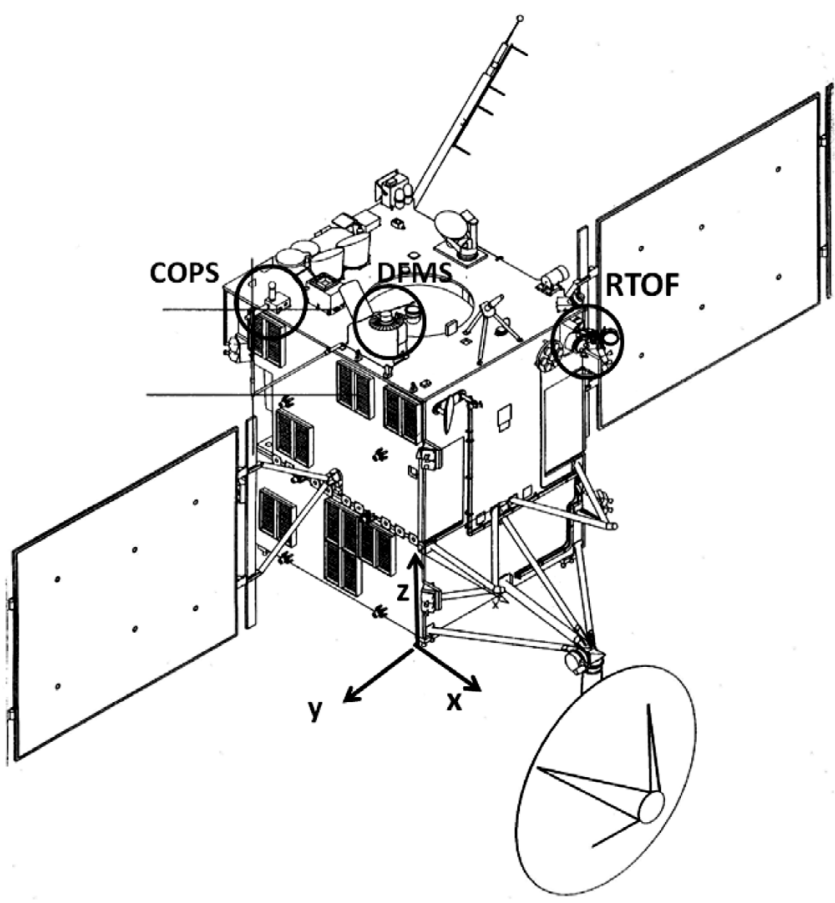

Figure 1. Rosetta spacecraft with the three ROSINA sensors (COPS, DFMS, and RTOF). The $+z$ panel (instrument panel) is on top. The lander is on the $-x$ panel. The solar aspect angle is measured between the spacecraft-Sun vector and $+z$ axis. In almost all cases, the solar aspect angle between the $y$ and $x$ axes is $90^{\circ}$ (perpendicular to the solar cells).

any direct line of sight to parts of the spacecraft. The field of view of the COPS nude gauge is almost $4 \pi$, and the field of view of the COPS ram gauge is $2 \pi$ centered on the $+\mathrm{z}$ axis. The nude gauge is mounted on a boom so that it will be in the free-flowing neutral gas from the comet.

[11] COPS measures total neutral density with the nude gauge and ram pressure with the ram gauge. The values are converted onboard with a reference temperature of $20^{\circ} \mathrm{C}$ into pressure. Lower limits are $10^{-11} \mathrm{mbar}$ for the nude gauge and $10^{-9} \mathrm{mbar}$ for the ram gauge. These lower limits are set by electrometer sensitivities. DFMS is a double focusing mass spectrometer with high mass resolution $(\mathrm{m} / \Delta m \sim 9000$ at full width half maximum) and sensitivity of $0.5 \mathrm{mbar}^{-1}$. RTOF is a time-of-flight mass spectrometer covering a large mass range. DFMS and RTOF were built to stringent ultrahigh vacuum specifications and were launched under vacuum. Their vacuum-tight covers were released approximately 2 months after launch. Their ion sources were baked up to $300^{\circ} \mathrm{C}$ in flight. DFMS and COPS have been operated several days per year since shortly after launch whereas, because of outgassing in the high-voltage electronics, RTOF was not operated between 2005 and February 2010 and is currently operated with reduced high voltages on the ion optical elements, resulting in somewhat lower sensitivity.

\section{Pressure Dependence on Elapsed Time and Heliocentric Distance}

[12] The COPS nude gauge, which is located on a boom extending about $25 \mathrm{~cm}$ from the instrument platform edge (in $\mathrm{Y}$ direction), measures total particle density. It was first turned on 20 days after launch and has been operated regularly since then. The COPS pressure as a function of elapsed time since launch is shown in Figure 2a. The first pressure reading, 20 days after launch and after allowing for the nude gauge to outgas, was at $2 \cdot 10^{-9}$ mbar, rapidly decreasing in subsequent months to $2.5 \cdot 10^{-10}$ mbar. After about 100 days after launch, the slope of the log of the pressure versus time changes noticeably (Figure 2a). Around 1100 days after launch, the COPS pressure measurements are close to the detection limit and therefore show large error bars. At that time, we can deduce the pressure from DFMS, which is far more sensitive, and find it identical to the COPS pressure within the measurement errors. During the first 100 days, Rosetta flew within 1 AU from the Sun. One year after launch, Rosetta's first Earth gravity assist occurred. The second Earth gravity assist took place approximately 1000 days after launch. Between these two periods, the heliocentric distance of Rosetta increased to 1.8 AU. In September 2008, Rosetta was even further away from the Sun (i.e., 2.2 AU) (compare Figure 2b). However, pressure depends very little on heliocentric distance of the Rosetta spacecraft. Tribble [2000] showed that three different mechanisms are responsible for the outgassing process. They all have different time dependencies and need different activation energies. The processes all follow the relation $e^{-\frac{E_{a}}{R T}}$, where $E_{a}$ is the characteristic activation energy, $R$ is the gas constant, and $T$ is the temperature. The lowest activation energy is needed by desorption from the surfaces of the spacecraft (4-40 kJ/mol [Tribble, 2000]). This corresponds to a $1 / e$ temperature range $\left(T=E_{a} / R\right)$ of $500-5000 \mathrm{~K}$ depending on the material. Desorption has an exponential dependence on time, and it also depends strongly on the temperature of the surfaces. This first mechanism is responsible for a pressure profile measured by COPS during the first 200 days after launch. This profile has a 1/e decay time of $30 \mathrm{~d}$ (Figure 2, dotted line). Rosetta was at a nearly constant heliocentric distance of about $1 \mathrm{AU}$ during this first period. Therefore, its surface temperature was relatively constant, and temperature dependence of desorption is not evident in the data.

[13] The second outgassing mechanism is diffusion. Gas from the spacecraft interior and from within the material diffuses slowly to the outside with a time dependence of $t^{-0.5}$ (Figure 2a, dashed-dotted line). The mean activation energy for diffusion is higher $(20-60 \mathrm{~kJ} / \mathrm{mol}$ [Tribble, 2000]) than that for desorption. As a result, the 1/e temperature range is $2500-7500 \mathrm{~K}$. Although the heliocentric distance of Rosetta changed by a factor $>2$, the surface temperature changed by not more than $50 \mathrm{~K}$, and the interior temperature changed by not more than $20 \mathrm{~K}$. Thus, for Rosetta, temperature has little influence on the diffusion process. The third mechanism arises from decomposition of material and is almost time independent (Figure 2a, dashed line). The activation energy is high $(80-320 \mathrm{~kJ} / \mathrm{mol})$. This activation energy can be supplied by, for example, solar UV, at least for Sun-exposed outer layers of the spacecraft.

[14] Measurements from the COPS pressure sensor and densities derived from DFMS can be very well understood by a combination of these three outgassing mechanisms (Figure 2). The contribution of decomposition to total outgassing is negligible for the first 1000 days after launch and 


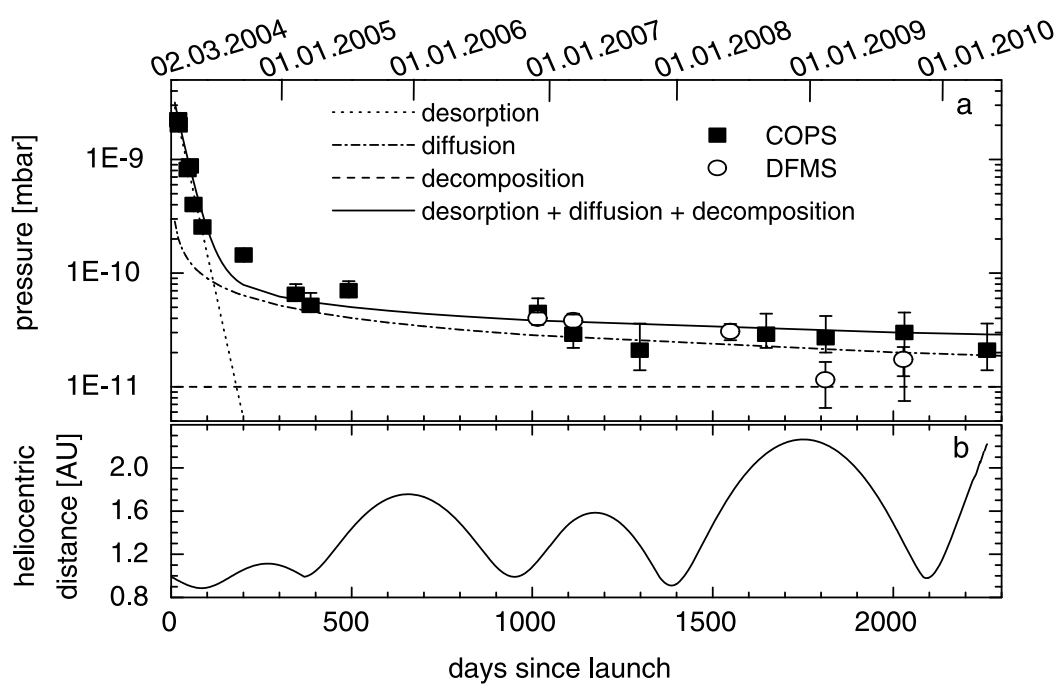

Figure 2. (a) COPS and DFMS pressure data as a function of time $t$ since launch. The dotted line shows the outgassing caused by desorption $p_{d} \propto e^{-t / t_{d}}$ with $t_{d}=30 \mathrm{~d}$. The dashed-dotted line is the outgassing attributed to diffusion $p_{d i f} \propto \frac{1}{\sqrt{t}}$, and the dashed line shows the contribution from decomposition $\left(p_{d c}=\right.$ const). The solid line represents the sum of the three contributions and has been fitted with the free parameters $\left(t_{d}, p_{d}, p_{d c}, p_{d i f}\right)$ to the data. Error bars of COPS measurements are uncertainties of digitalization, whereas error bars of DFMS measurements are attributed to ion statistics. (b) Heliocentric distance of Rosetta as a function of time since launch.

still less than 50\% after 2000 days after launch. Extrapolating the curve to 4000 days after launch, when Rosetta will be at the comet, the pressure will be still above $10^{-11} \mathrm{mbar}$ and will be still governed by diffusion.

[15] Pressure history of weekly recorded measurements of the TPS instrument on the MSX mission [Uy et al., 2003] also showed expected exponential decay during the first months of mission lifetime, presumably because of desorption of mainly water. Comparing TPS measurements with the Rosetta data (Figure 3), the time constants of exponential decay for both instruments within the first 100 days are very similar. Deduced 1/e decay times are 30.7 days for the TPS instrument and 30.1 days for the COPS instrument, respectively, resulting in a nearly parallel slope of both data sets. This is not surprising as both spacecraft were at 1 AU heliocentric distance and desorption dominated early in the missions.

\section{Composition of the Gas Cloud Around the Spacecraft}

[16] DFMS is operated in low- and high-resolution modes, covering a mass range between 12 and $>100$ amu. A lowresolution spectrum taken in March 2010 at 1.7 AU is shown in Figure 4. The inset shows part of the mass spectrum on a linear scale. The particle density given on the $y$ axis has to be interpreted with caution, taking into account the uncertainty of the calibration. Different detector yields and ionization cross sections have not been taken into account fully. The error may be as much as a factor of 5 . As can easily be seen in Figure 4, there is a signal for almost every integer mass number.

[17] In the DFMS spectra, the dominant mass is water (at $18 \mathrm{amu} / \mathrm{e})$ with a density of about $5 \cdot 10^{5} \mathrm{~cm}^{-3}(T=150 \mathrm{~K})$. There is a rich variety of organic compounds present with densities $\leq 10^{4} \mathrm{~cm}^{-3}$. This compares well with estimates from the SOHO spacecraft for water [Defise et al., 1997] and for organic compounds [McMullin et al., 2002].

[18] In high-resolution mode, parent molecules and fragments can be identified. Figure 5 shows DFMS highresolution spectra as examples for two masslines. From the high-resolution spectra over the full mass range (12$130 \mathrm{amu} / \mathrm{e})$, we derived all the species and fragments compiled in Figure 6.

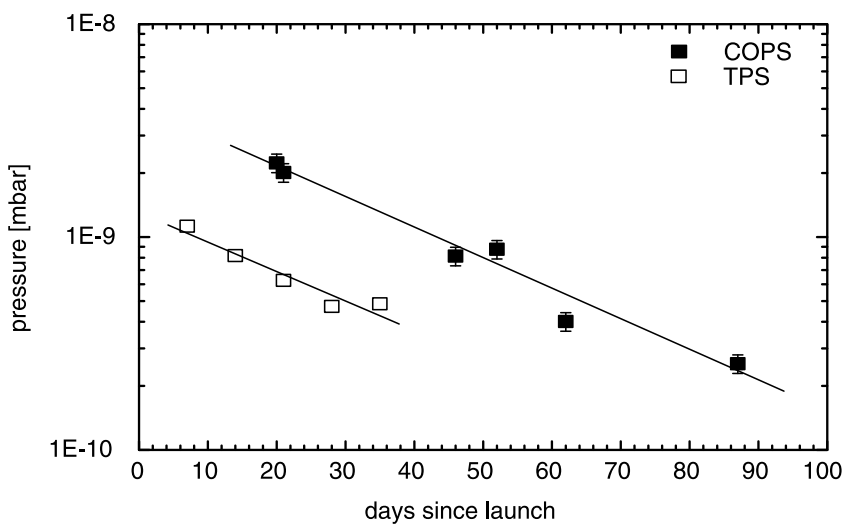

Figure 3. A comparison of the pressure data from the TPS sensor on the MSX spacecraft [Uy et al., 2003] and ROSINA-COPS on Rosetta for the first 100 days of the mission. Lines are exponential fits to the data. The TPS sensor was about a factor of 20 less sensitive than the COPS sensor. Therefore, the data stop at $2 \cdot 10^{-10} \mathrm{mbar}$, which was reached after 40 days. The pressure on Rosetta is about a factor of 5 larger, probably because of the much bigger size of the spacecraft. The $1 /$ e decay time of desorption $\left(t_{1 / e}=30\right.$ days $)$, however, is almost identical for the two spacecraft. 


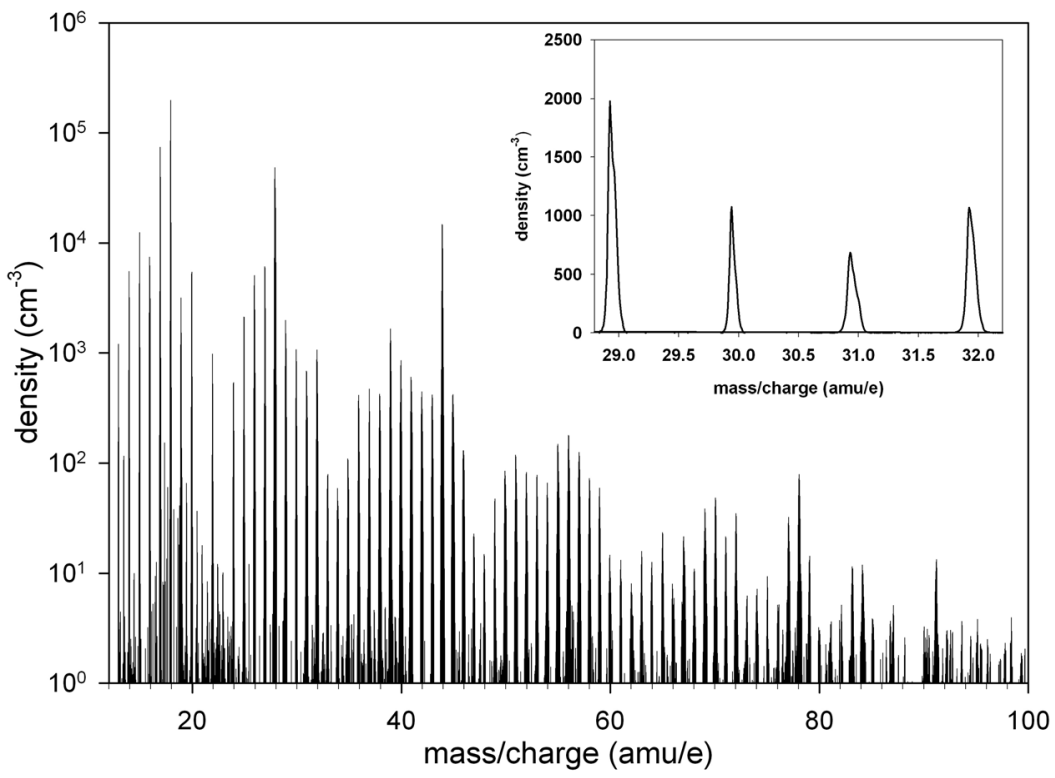

Figure 4. DFMS mass spectrum in low resolution of 14 March 2010, at a heliocentric distance of 1.7 AU. Integration time per mass line is $20 \mathrm{~s}$, and electron emission current is $200 \mu \mathrm{A}$. The inset shows part of the mass spectrum in a linear scale to illustrate the mass resolution in this low-resolution mode.

[19] In the RTOF spectra, the dominant mass is fluorine (at mass $19 \mathrm{amu} / \mathrm{e}$ ) with a density of about $2 \cdot 10^{6} \mathrm{~cm}^{-3}$; water is about the same as in the DFMS spectra. Again, we find a variety of organic compounds in the spectra. The $\mathrm{H}$ and $\mathrm{H}_{2}$ lines are mostly fragments of the hydrocarbon compounds, but also from the parent molecule of the fluorine.

[20] It is evident that hydrocarbons are abundant. In addition, many nitrogen-bearing molecules are found, some of them with oxygen complexes. Also, halogen- and sulfurcontaining compounds are identified.

\subsection{Hydrocarbon Compounds}

[21] Hydrocarbon molecules and polycyclic-aromatic hydrocarbon molecules have been identified in ROSINA mass spectra. Possible sources for hydrocarbons are, of course, polycarbonates (from the spacecraft structure) and solvents. Toluene, xylene, and other solvents or fragments thereof have been identified.

\subsection{Nitrogen-Bearing Compounds}

[22] Among them are the parent and fragments of monomethylhydrazine, the propellant of the Rosetta spacecraft. However, the amount and distribution of these nitrogenbearing fragments suggest other sources that are probably much stronger than thruster fuels. Common spacecraft contamination sources are polyurethane (structure, conformal coatings, and adhesives), epoxies (structure, potting, conformal coatings), polyamines (structure), and polyimides (structure) [Tribble, 2000].

\subsection{Halogen- and Sulfur-Bearing Molecules}

[23] Fluorine is often observed in mass spectrometers with filament ionization sources (e.g., for the mass spectrometer for the Pioneer Venus mission) [McFadden et al., 2007]. Possible sources for fluorine are remnants from brazing, fluorocarbons contained in the structure, in tapes, and in lubricants. DFMS and RTOF both showed traces of fluorine prior to launch. On the contrary, chlorine and sulfur were not detected prior to launch; however, they may have many sources because they are known contaminants in a lot of processes and are also contained in some solvents.
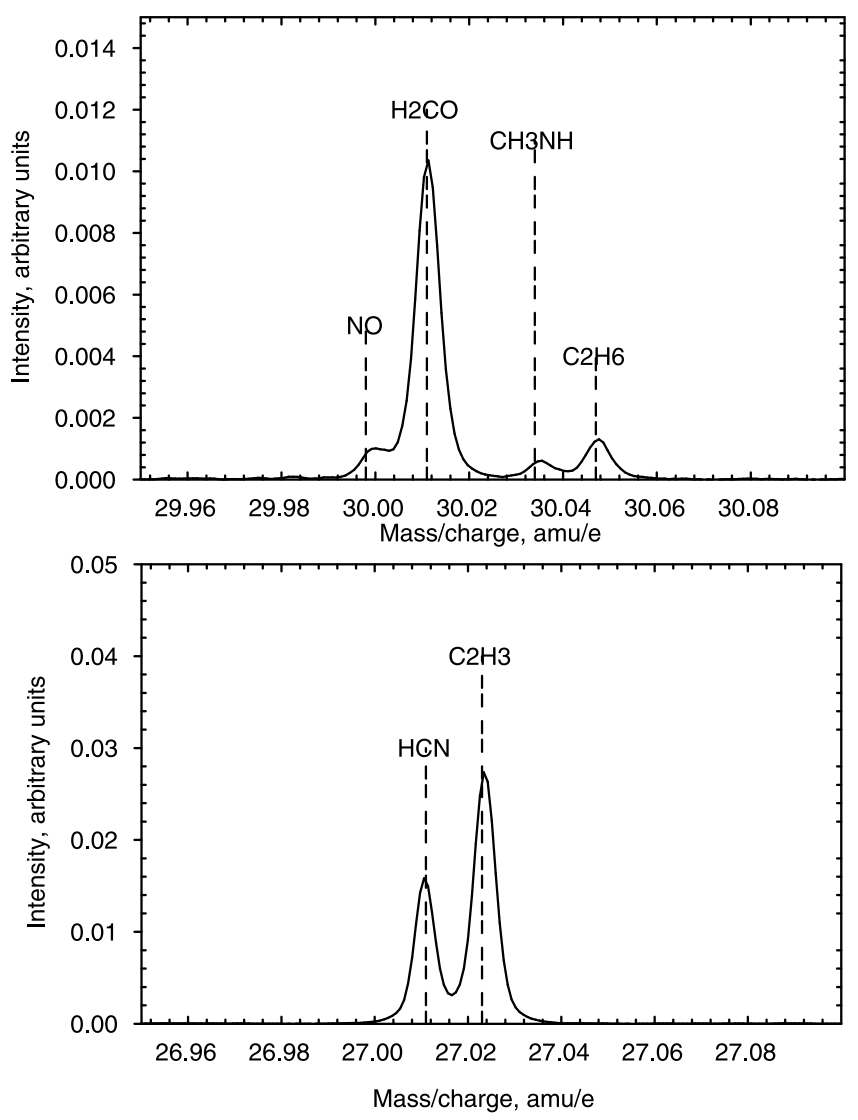

Figure 5. Two DFMS high-resolution spectra. Integration time is $20 \mathrm{~s}$, and electron emission current is $200 \mu \mathrm{A}$. 


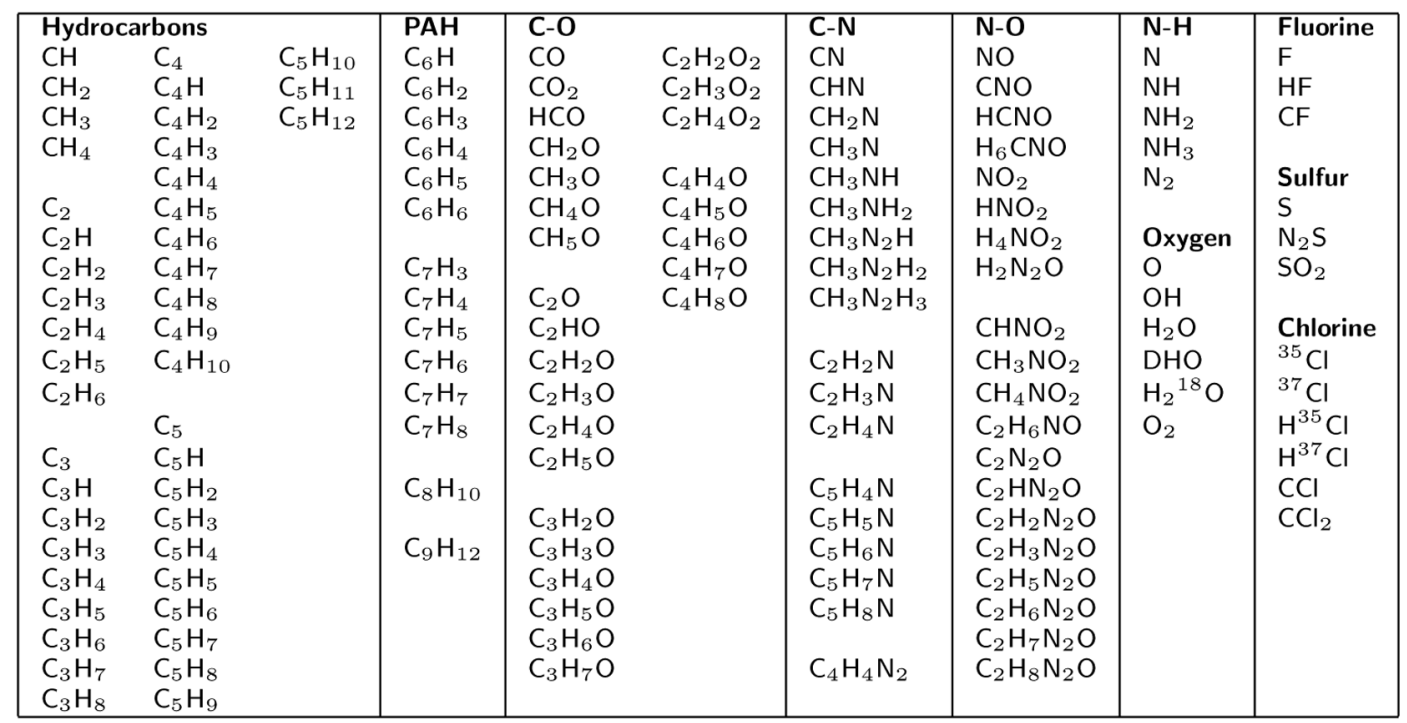

Figure 6. Detected species and fragments in the vicinity of Rosetta.

[24] Heating the DFMS ion source for several days at $300^{\circ} \mathrm{C}$ in flight neither changed the amount of gas measured nor its composition. That means, except part of the fluorine, that recorded chemicals are not from the DFMS ion source but are from the gas cloud around the Rosetta spacecraft.

\subsection{Lubricant}

[25] A comparison between a RTOF mass spectrum and a low-resolution DFMS spectrum, both taken in March 2010, reveals significant differences (Figure 7). For DFMS, the highest peak is clearly water at mass/charge $18 \mathrm{amu} / \mathrm{e}$ followed by mass $28\left(\mathrm{CO} / \mathrm{N}_{2}\right)$ and mass $44\left(\mathrm{CO}_{2}\right)$. For RTOF, the highest peak is fluorine, which is more than a factor of 10 larger than the water peak. Apart from this peak, mass 15 $\left(\mathrm{CH}_{3}, \mathrm{NH}\right)$, the group around mass 28 with significant peaks at 27 and 29, and the group around mass 40 with significant peaks at 39, 41, and 43 are prominent, whereas water and $\mathrm{CO}_{2}$ are rather minor. The pattern in the RTOF spectrum around mass 28 and $40 \mathrm{amu} / \mathrm{e}$ is very typical for aliphatic (C-C bonds) compounds. Interestingly, Marshall et al. [2004] deduced the same pattern with the Advanced CCD Imaging Spectrometer (ACIS) on the Chandra X-ray mission. The authors analyzed the contaminant of this instrument by the X-ray absorption edges and determined the composition of the contaminant to be $>80 \%$ carbon, $7 \%$ oxygen, and $7 \%$ fluorine. They concluded that there are no $\mathrm{C}=\mathrm{C}$ bonds and no $\mathrm{C}-\mathrm{F}$ bonds but that the contaminant has to be an aliphatic fluorine-containing molecule. These facts point to the lubricant (Braycote vacuum grease) for the source of the contamination in the case of ACIS/Chandra. This lubricant is used in different mechanisms of Chandra. Braycote is a perfluorated polyester that can be damaged by radiation (UV, X-ray) and be converted into an aliphatic compound. The high gain antenna of Rosetta uses the same lubricant. The antenna is attached to the $+x$ panel (Figure 1). The $+\mathrm{x}$ panel is normally in sunlight, which may lead to relatively high temperatures and to a degradation of the lubricant. The difference between RTOF and DFMS can be explained by their different locations on the spacecraft. The
RTOF ion source is on the same panel as the high gain antenna and not too far away. The view direction is the same as for DFMS in the direction of $+z$. DFMS is on the $+z$ panel, together with the rest of the payload. Although there is no direct line of sight between the entrance of the RTOF ion sources and the high gain antenna mechanisms, gas can much more easily be scattered into the RTOF ion sources than into the DFMS ion source. Still, some of the degraded lubricant also reaches the entrance of DFMS as can be seen by the fluorine peak. We can therefore confirm the findings of the ACIS/Chandra contamination investigation that the lubricant Braycote forms aliphatic compounds under radiation that are more volatile than the original molecules.

\section{Variability of Background}

[26] During the past year, a set of data was acquired by ROSINA, indicating that the background as discussed in section 4 is not constant with time, not even on a short time scale. We find that several operational effects are influencing instrument contamination and background in the mass spectrometers must be taken into account.

\subsection{Spacecraft Attitude}

[27] Since instruments are, in general, fixed with respect to the spacecraft coordinate system, the spacecraft has to change attitude to insure an optimal view direction for the payload. Optimizing the orientation results in changing illumination conditions of countless parts of a spacecraft, in particular exposing panels to sunlight that have been in shadow earlier, and vice versa. This effect was already reported by Uy et al. [2003] for the MSX satellite. We demonstrate that this effect constrains scientific measurements at larger heliocentric distances.

\subsubsection{Asteroid Steins Flyby}

[28] The effect of spacecraft attitude on the ROSINA instrument background was recognized during ESA's first asteroid flyby in September 2008: Rosetta approached its target, the E-type asteroid (2867) Steins, with a relative 


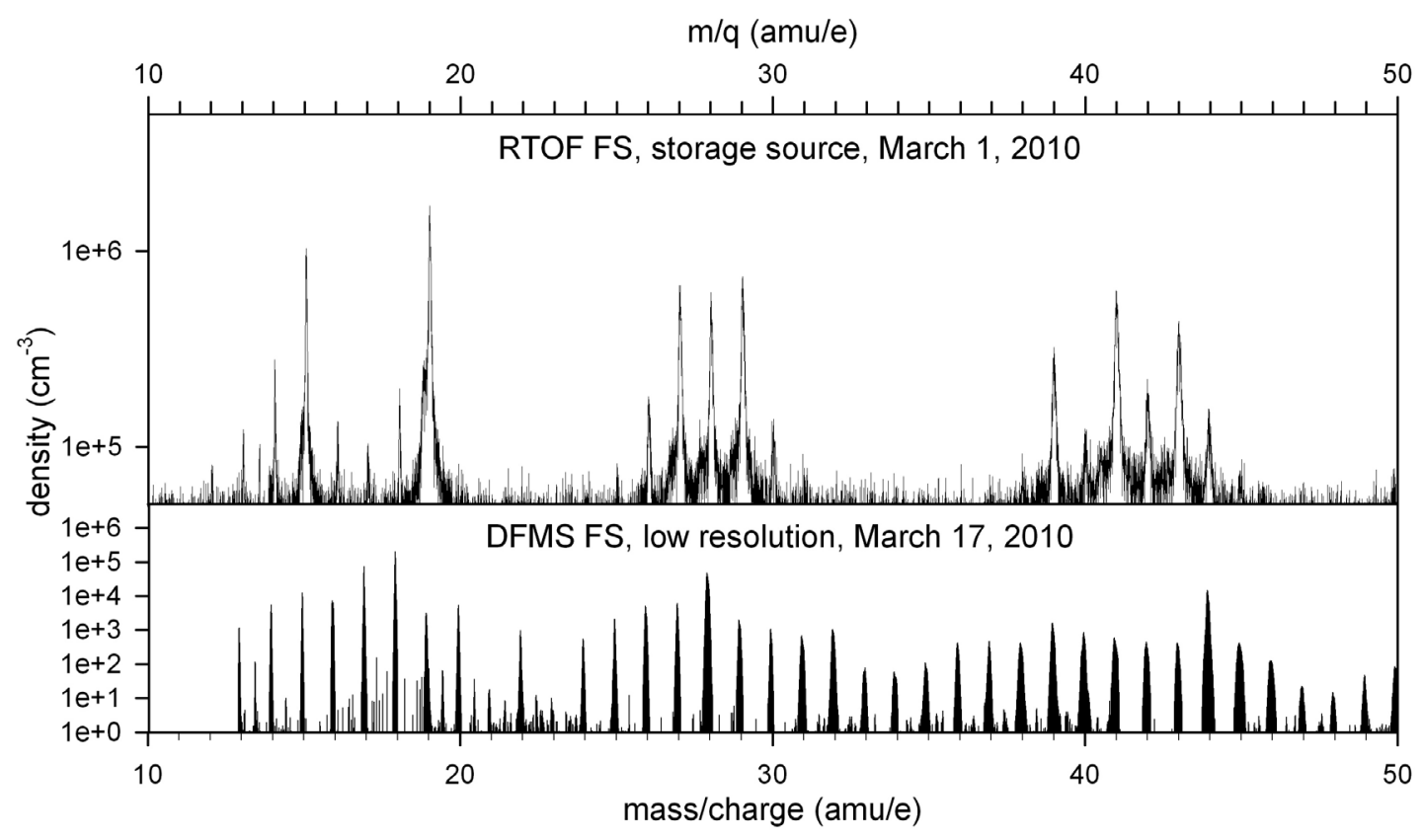

Figure 7. Comparison of a low-mass section of mass spectra from DFMS and RTOF taken in March 2010. The RTOF peak at mass 19 is fluorine, which is partly from an internal source. The acquisition time of DFMS for the total spectrum was 20 min compared to 3 min for RTOF, and the DFMS electron emission was higher by a factor of 2, which accounts partly for the higher DFMS sensitivity.

velocity of $8.6 \mathrm{~km} / \mathrm{s}$ to approximately $800 \mathrm{~km}$ closest approach (CA) on 5 September 2008 at 1838:20 UTC. The flyby took place at a heliocentric distance of $2.1 \mathrm{AU}$.

[29] Two spacecraft maneuvers were performed during the flyby. From 1758 to 1818 UTC, Rosetta rotated around its $z$ axis (Figure 1) to prevent illumination of thermally sensitive parts, which would have limited observations after $\mathrm{CA}$. The second maneuver was a slew started shortly before CA to keep the $z$ axis (bore sight of instrument platform) pointing to the asteroid and allow continuous observation by remote sensing instruments.

[30] Planned ROSINA measurements during the Steins flyby were intended to contribute to exploration of the asteroid's environment, in particular to search for a lowlevel gaseous envelope [Schläppi et al., 2008]. The flyby strategy was to use COPS to monitor total neutral particle density, while DFMS would monitor a narrow mass range. DFMS focused on a limited mass range to avoid timeconsuming adjustments of the ion optical potentials and thus risk missing interesting data during the short flyby. Since (2867) Steins is a thermally evolved object, the monitored mass was set to detect sputtered oxygen from the asteroid's surface; the monitored mass to charge ratio was set to $\mathrm{m} / \mathrm{q}=$ $15.5 \mathrm{amu} / \mathrm{e}$, which allows simultaneous detection of several species: $\mathrm{O}, \mathrm{CH}_{4}, \mathrm{NH}_{2}$ as well as $\mathrm{CH}_{3}$ and $\mathrm{NH}$.

[31] ROSINA results from the Steins flyby are presented in Figure 8 . Figure 8 clearly shows a correlation between spacecraft attitude and instrument background. This led to our hypothesis that illumination of cold panels exposed to sunlight after years in darkness causes evaporation of gases condensed on these panels.

[32] One of the peculiarities that attract attention in Figure 8 is the time delay of signal variations observed between DFMS and COPS. The spatial separation of the sensors of about $1 \mathrm{~m}$ and typical velocities of thermally released particles (a few times $100 \mathrm{~m} / \mathrm{s}$ ) are certainly not the reason for the observed time delay of a few minutes. To explain this delay, one needs to consider different FOV of the two sensors (Figure 1). It is therefore reasonable to assume that certain outbursts from the spacecraft are observed by COPS, but not by DFMS. However, the first peak observed by DFMS is also observed in COPS data with a partial pressure of approximately $10^{-10} \mathrm{mbar}$. This is evident in the slope of the exponential decay around 1817 UTC, which changes because of the outburst observed by DFMS.

[33] We exclude the possibility that the observed variations are due to internal instrument outgassing since during the first DFMS pressure peak (maximum at $\sim 1820$ UTC), the ion source was not illuminated at all and the temperature increase observed is negligible (from $-26.8^{\circ} \mathrm{C}$ at $1800 \mathrm{UTC}$ to $-25.6^{\circ} \mathrm{C}$ at $1820 \mathrm{UTC}$ ). Furthermore, the ion source was baked out at $300^{\circ} \mathrm{C}$ for almost 2 days preceding these measurements.

\subsubsection{Compositional Measurements During Spacecraft} Thermal Characterization Tests

[34] Based on results from the Steins flyby, the European Space Operation Control team provided ROSINA with an additional opportunity to investigate the observed behavior: during a spacecraft thermal characterization test conducted 16-18 February 2009, ROSINA was able to perform extended compositional measurements of the spacecraft desorption behavior.

[35] Characterization tests consisted of several test periods at different orientations. On 16 February 2009 at 1400 UTC, Rosetta was slewed from its "nominal" attitude, with SAA $\sim 72^{\circ}$, to SAA $=175^{\circ}$. During the entire test period, the S/C $y$ axis was perpendicular to the Rosetta-Sun axis. Rosetta remained at this orientation for $24 \mathrm{~h}$ and was then slewed by 

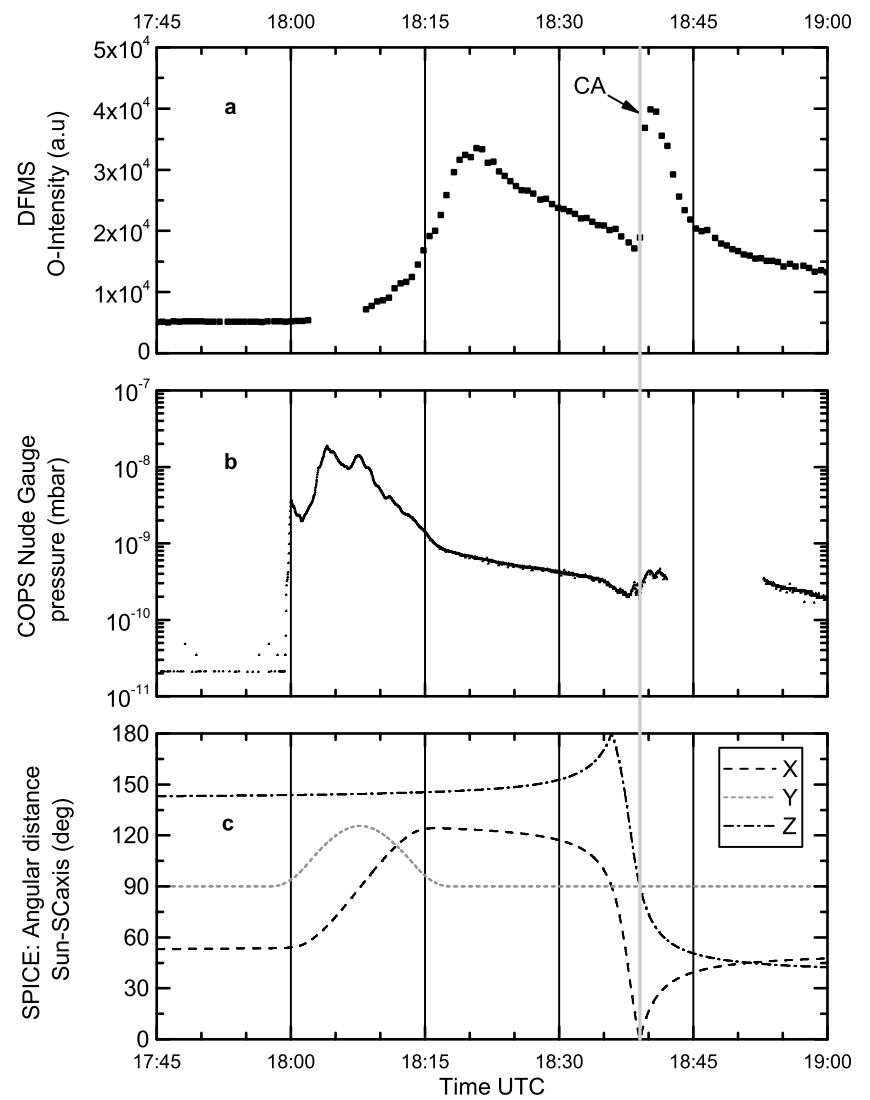

Figure 8. ROSINA Steins flyby results from COPS and DFMS. (a) Two peaks were observed in the DFMS oxygen intensity. One clearly correlated with the S/C flip, and the other one appeared around CA (indicated by bold line). (b) COPS nude gauge pressure with a sharp edge when the flip was started (1758 UTC). Note the change in the slope around 1817 UTC. Variations are also apparent around CA. (c) Rosetta spacecraft orientation according to SPICE [Acton, 1996].

$17^{\circ}$. With SAA $=192^{\circ}$, the spacecraft $-x$ panel was slightly illuminated. After another $24 \mathrm{~h}$, Rosetta returned to the initial orientation of SAA $=72^{\circ}$. The maneuvers took place at a heliocentric distance of approximately 2.2 AU. Figure 9 depicts total pressure during the characterization as measured by the COPS nude gauge. Obviously, during every maneuver, COPS observes pressure peaks, corresponding to release of volatiles from the spacecraft. Surprisingly, adjacent to the slew on 17 February at 1400 UTC, the pressure remains at least one order of magnitude higher than before the slew (considering the lower detection limit of about $1 \cdot 10^{-11}$ mbar of COPS).

[36] Thermal characterization tests, in particular the maneuvers on 17 February, were used to investigate the composition of the spacecraft outgassing. Several low- and high-resolution spectra over the full mass range of DFMS $(m / q=12-140 \mathrm{amu} / \mathrm{e})$ were taken before and after the slew. During the maneuver, the same mode as during the Steins flyby was used. Figure 10 shows that water clearly dominates the released gas, but small amounts of $\mathrm{CH}_{4}$ and $\mathrm{CO}_{2}$ might be present.
[37] With current instrument sensitivities, we estimate that the partial $\mathrm{H}_{2} \mathrm{O}$ pressure increased from approximately $2.3 \cdot 10^{-11}$ mbar (approximate particle density of 1.1 . $\left.10^{6} \mathrm{~cm}^{-3}\right)$ at 1230 UTC to $6.1 \cdot 10^{-11} \operatorname{mbar}\left(2.9 \cdot 10^{6} \mathrm{~cm}^{-3}\right)$ at 1445 UTC, while the partial $\mathrm{CO}_{2}$ pressure was 3.8 . $10^{-12}$ mbar $\left(1.8 \cdot 10^{5} \mathrm{~cm}^{-3}\right)$ before and $4.7 \cdot 10^{-12} \mathrm{mbar}$ $\left(2.3 \cdot 10^{5} \mathrm{~cm}^{-3}\right)$ after the slew. COPS observed an increase in the total pressure by a factor 10 , in contrast to DFMS, where only the partial water pressure was increased by a factor of approximately 3 . The observed difference might be a superposition of the already described field of view effect and the proximity of the sensors with respect to the source of the released gas. Furthermore, derived partial pressures suffer from large uncertainties (approximately 50\%) since DFMS is still not optimized and no extensive, quantitative calibration has been performed for water yet. Despite these uncertainties, results indicate a significant water reservoir, possibly carried along with Rosetta since launch in 2004 and replenished by combustion of monomethylhydrazine during thruster firings, which yields $\mathrm{H}_{2} \mathrm{O}$ and $\mathrm{CO}_{2}$ [de Bonn et al., 2001].

\subsection{Payload Operations}

[38] In most cases, space probes carry a set of different instruments, each contributing to the main scientific goals of the mission. It is clear that during periods where science data are recorded, several instruments operate simultaneously and therefore dissipate energy and warm up. As they warm, they contribute to background gas. This effect was observed by COPS several times when the Grain Impact Analyzer and Dust Accumulator instrument [Colangeli et al., 2007]) was operated simultaneously. COPS registered an increased background pressure (approximately factor 4 ) during these interference tests. However, this increase is not surprising since the Grain Impact Analyzer and Dust Accumulator is one of the closest instruments to COPS. More staggering are measurements performed during a Rosetta payload checkout in September 2009, in which several interference tests or combined measurements with other payload instruments have been performed in conjunction with DFMS operation.

[39] Figure 11 shows results of an interference test with the Rosetta lander PHILAE [Bibring et al., 2007] as well as an attempt to combine measurements with Ptolemy, a gas chromatograph mass spectrometry sensor onboard the lander [Wright et al., 2007]. Both units are mounted on the spacecraft $-x$ panel and are therefore clearly outside the DFMS FOV. During the lander interference test, a clear increase in $\mathrm{H}_{2} \mathrm{O}$ and $\mathrm{CO}_{2}$ signals was observed by DFMS as soon as the lander was operated.

[40] Another interference test was planned with Ptolemy. The plan was to cross-calibrate the two mass spectrometers (DFMS and Ptolemy). Unfortunately, these measurements could not be conducted because Ptolemy suffered an unexpected anomaly. But, as Figure 11 shows, DFMS detected an increase in the partial pressure of water as soon as Ptolemy was switched on.

[41] During both interference tests, the spacecraft attitude remained constant, no ROSINA instrument anomalies occurred, and no variations of sensor temperatures could explain the increase in partial pressures. The results of these tests provide strong indication that instrument operations must be considered to assess variability of the background. 


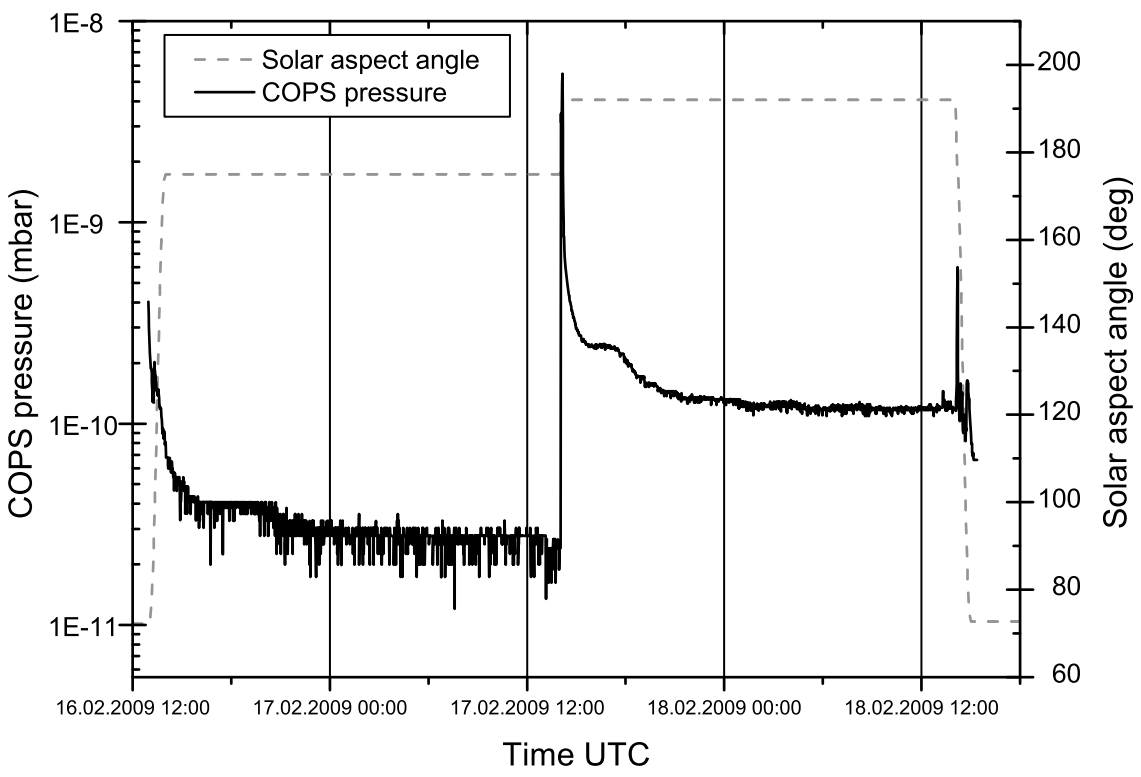

Figure 9. Solar aspect angle and COPS nude gauge pressure during the thermal characterization test of Rosetta in February 2009. The first peak observed by COPS is attributed to internal outgassing, but an overlapping peak is visible when SAA was approximately $90^{\circ}$.

\subsection{Thruster Operations}

[42] It has been shown elsewhere that thruster operations can lead to a large increase in pressure around the spacecraft [Graf et al., 2008]. While pressure increases attributed to thruster operation can be even harmful for instruments (mainly increasing the danger of discharge for instruments using high voltages), the pressure after a thruster firing decreases relatively rapidly within minutes to acceptable levels for payload integrity. There remains, however, a higher pressure for tens of minutes after the thruster firing at

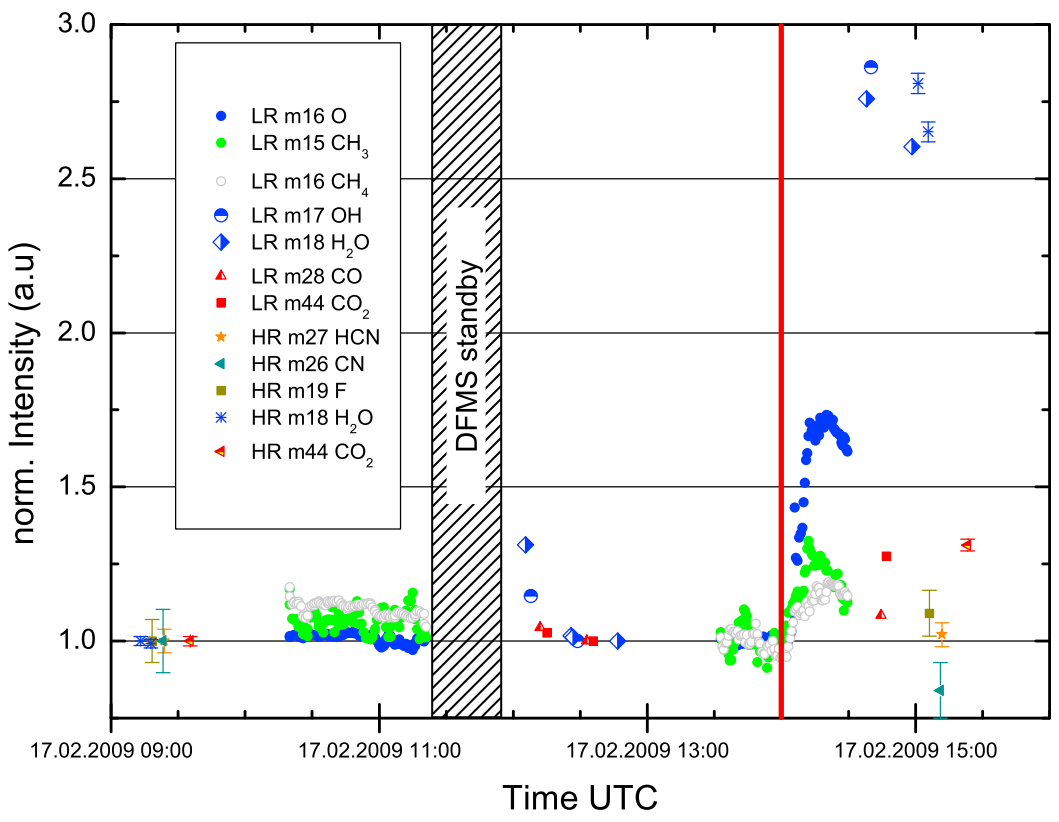

Figure 10. DFMS composition measurements during the slew of 17 February 2009. Several lowresolution (LR) and high-resolution (HR) spectra were obtained before and after the maneuver, and the intensities have been normalized to the last spectrum obtained before 1345 UTC. The red line indicates the slew. From approximately 1125 to 1152 UTC, DFMS was set to standby to prevent potential software complications. This explains the small peak at $~ 1200$ UTC, when the filament was heated again and some outgassing from the filament occurred. Error bars from ion statistics were only drawn for high-resolution spectra; for others, they are negligible. The instrument performance was without any problems, and DFMS was in shadow during the entire maneuver. We therefore exclude outgassing of the sensor itself and conclude that the observed outburst was a result of the illumination condition. 

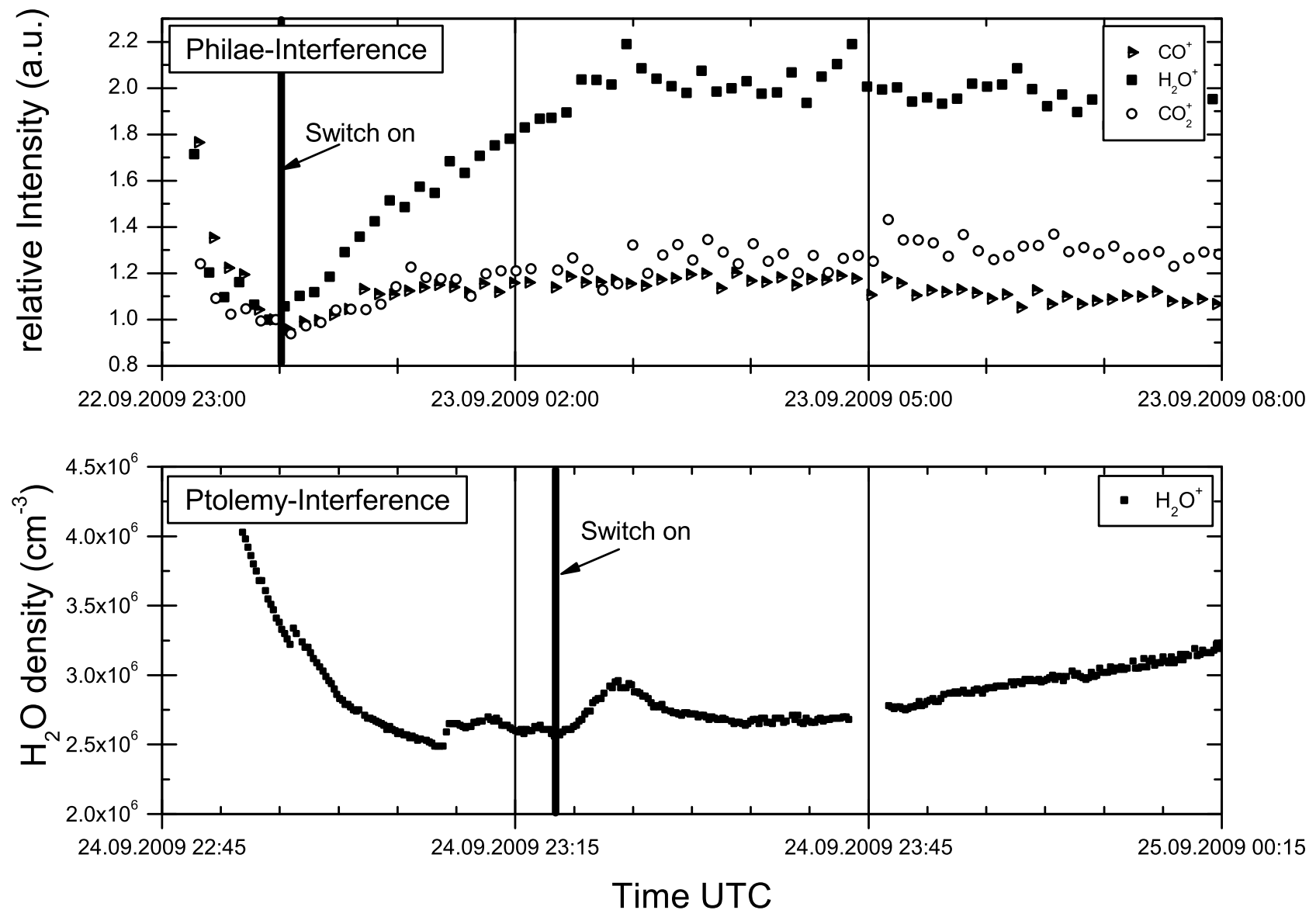

Figure 11. Results from interference tests between ROSINA and (top) the lander Philae and (bottom) Ptolemy. The thick line indicates the switch on of the corresponding unit. The flank of the signal at the beginning of each measurement is attributed to DFMS outgassing of the filament.

the position of the COPS nude pressure sensor despite the shielding of the direct line of sight between COPS and all thrusters. Figure 12 shows the total pressure for more than 3 days starting before and proceeding through the Steins flyby. There are several large pressure peaks. Peaks labeled with TF1, TF2, and TF3 are due to thruster firing of various thrusters. The peak that is not labeled is due to the spacecraft flip of almost $180^{\circ}$ around the $y$ axis.

[43] Figure 13 shows details of the third thruster firing (TF3). There is clearly a fast drop of the pressure after, in this case, multiple thruster firings, but there remains an elevated pressure of about $2 \cdot 10^{-11}$ mbar for more than $1 \mathrm{~h}$. This elevated pressure may be due to hydrazine droplets evaporating slowly from the thruster nozzles (not very likely because of the shielding of the thrusters) or to evaporation of thruster firing products deposited on different spacecraft surfaces during thruster operation.

\section{Origin of the Gas Cloud Around Spacecraft}

[44] The observed deterioration of optical surfaces and the noninternal neutral background measured with mass spectrometers presented in this study require consideration of a redistribution of the contamination onboard a spacecraft, likely caused by a significant return flux of released material.
[45] For satellites in geosynchronous Earth orbit, Thomas et al. [1998] noted that the main return flux mechanism for neutral particles is molecular backscattering caused by collisions among released material. Robertson [1976] presented a simple model to estimate the relative return flow rate of outgassing products caused by collisions among outgassing molecules (self-scattering) and collisions among outgassing and ambient molecules (ambient scattering). Using this model and results from the Molecular Return Measurement Unit experiment onboard the Atmosphere Explorer D satellite, Scialdone et al. [1978] estimated that at altitudes of 300-350 km, self-scattering becomes the major contribution to the return flow in the Earth atmosphere. Ambient scattering is negligible for deep space missions, and we therefore use the self-scattering model of Robertson [1976] to estimate the release rate necessary to backscatter enough material to explain the measurements of the DFMS sensor: for a spherical spacecraft of radius $R$ with isotropic outgassing, the return flow fraction $\Phi_{b} / \Phi_{r}$, with $\Phi_{b}$ the backscattered flux and $\Phi_{r}$ the released flux (in units molecules $\mathrm{m}^{-2} \mathrm{~s}^{-1}$ ), can be calculated from the following expression (derived from Robertson [1976]):

$$
\frac{\phi_{b}}{\phi_{r}}=\left(\frac{0.01554-\frac{0.003596 \sigma m R \phi_{r}}{\sqrt{m k T}}}{\sqrt{m k T}}\right) 0.8862 \sigma m R \phi_{r},
$$




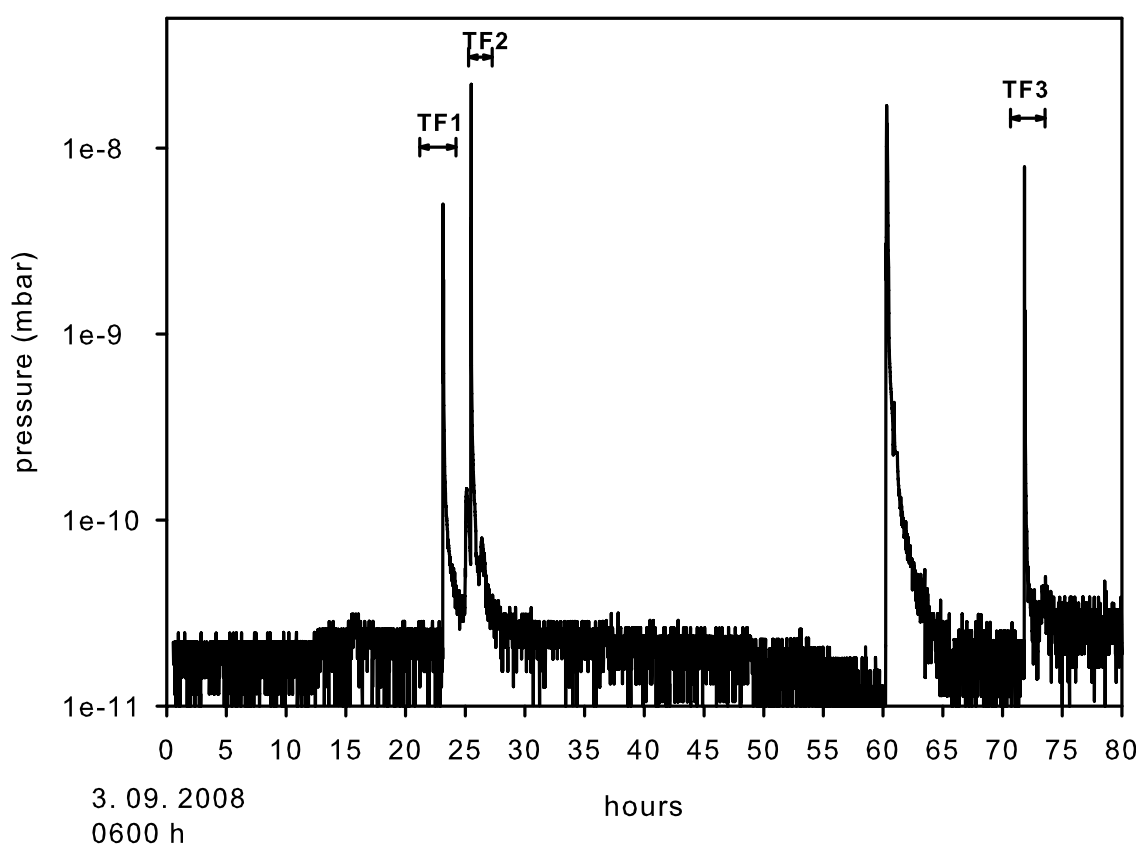

Figure 12. Total pressure measured by COPS during the asteroid Steins flyby. The peaks denoted by TF1, TF2, and TF3 are all coincident with thruster firings. The peak at $60 \mathrm{~h}$ after start of the measurement is caused by a spacecraft flip of $180^{\circ}$ around the $y$ axis.

where $m$ is the mass, $\sigma$ is the collisional cross section of the released particles, and $k$ is the Boltzmann constant. Using the observed particle number density $\left(10^{11} \mathrm{~m}^{-3}\right)$ and an assumed particle velocity of $500 \mathrm{~m} / \mathrm{s}$, we derive a backscatter flux of $\Phi_{b}=5 \cdot 10^{13} \mathrm{~m}^{-2} \mathrm{~s}^{-1}$ entering the ion source of the DFMS or RTOF instrument. Solving the above equation for the release rate with parameters of $\mathrm{H}_{2} \mathrm{O}$ molecules $\left(m=18 \mathrm{amu}, \sigma=2.5 \cdot 10^{-19} \mathrm{~m}^{2}\right)$, a spherical spacecraft with radius $R=1.5 \mathrm{~m}$ and a wall temperature of $T=300 \mathrm{~K}$ results in a (physical meaningful) water loss rate of about $1.9 \cdot 10^{18}$ molecules $\mathrm{m}^{-2} \mathrm{~s}^{-1}$ for the spherical spacecraft, which seems quite reasonable. This water loss rate corresponds to the release of 0.2 monolayers per second or to about $1.8 \mathrm{kgm}^{-2} \mathrm{yr}^{-1}$ for the spherical spacecraft. Contrary to this model, a real spacecraft is considerably more complex. The main part of the observed particle densities probably arises through direct flow and (multiple) scattering of contaminants on spacecraft parts. Therefore, we expect a significant lower release rate per unit area than obtained from the sphere model.

[46] However, the total release rate of molecules of Rosetta is on the order of several hundred grams per year. Rosetta has a dry mass of $1180 \mathrm{~kg}$. We assume that $30 \%$ of Rosetta consists of nonmetallic material (epoxy, carbon fiber structure, multilayer blankets, electronics, insulated wiring, etc.). This spacecraft material has been selected for its low outgassing, defined by its total mass loss of less than $1 \%$ at $125^{\circ} \mathrm{C}$ in $24 \mathrm{~h}$ in vacuum [Campbell and Scialdone, 1993]. The characteristic time constant for outgassing attributed to diffusion is indirectly proportional to the diffusion coefficient and therefore follows the relation $e^{-\frac{E_{a}}{R T}}$ (compare to chapter 3 for the notation). Hence, with a generic activation energy of $E_{a}=50 \mathrm{~kJ} / \mathrm{mol}$, time scales for outgassing at a temperature of $0^{\circ} \mathrm{C}$ (which is approximately what we expect for a spacecraft) is about a factor $10^{3}$ larger compared to the specifications at $125^{\circ} \mathrm{C}$. A material meeting the outgassing specification will therefore suffer approximately the same total mass loss during $1000 \mathrm{~d}$ in flight as during the outgassing tests. Considering the limit of $1 \%$ total mass loss during test periods, we conservatively estimate an average lifetime loss rate of $0.5 \%$. The estimated nonmetallic material onboard Rosetta would therefore lose about $2 \mathrm{~kg}$ or more during its 10 year mission duration. Additionally, all surfaces carry adsorbed material (water). An estimation of the corresponding active area is difficult, especially since certain spacecraft materials have significantly larger effective surfaces than geometric area (e.g., MLI or honeycomb structures). If we account only for the central box of Rosetta with a total surface of $31 \mathrm{~m}^{2}$ and the solar panels with a surface of $64 \mathrm{~m}^{2}$ (which are made from honeycomb structure), we get an effective surface area of about $5000 \mathrm{~m}^{2}$. The total surface area of Rosetta may well be up to $10000 \mathrm{~m}^{2}$. Adsorbed water on aluminum surfaces of the honeycomb structure is on the order of $10^{21}$ molecules $\mathrm{m}^{-2}$ [Chen et al., 2001], which yields several hundred grams of water eventually diffusing to the outside. The reservoir for outgassing of water and organic compounds is therefore quite large and can easily explain observed densities.

\section{Conclusions}

[47] The most important process of outgassing during the first 200 days of a mission is desorption of water from exposed surfaces of the spacecraft. The half-life of the density associated with desorption is approximately 21 days for a spacecraft at $1 \mathrm{AU}$. Prolonged outgassing caused by 


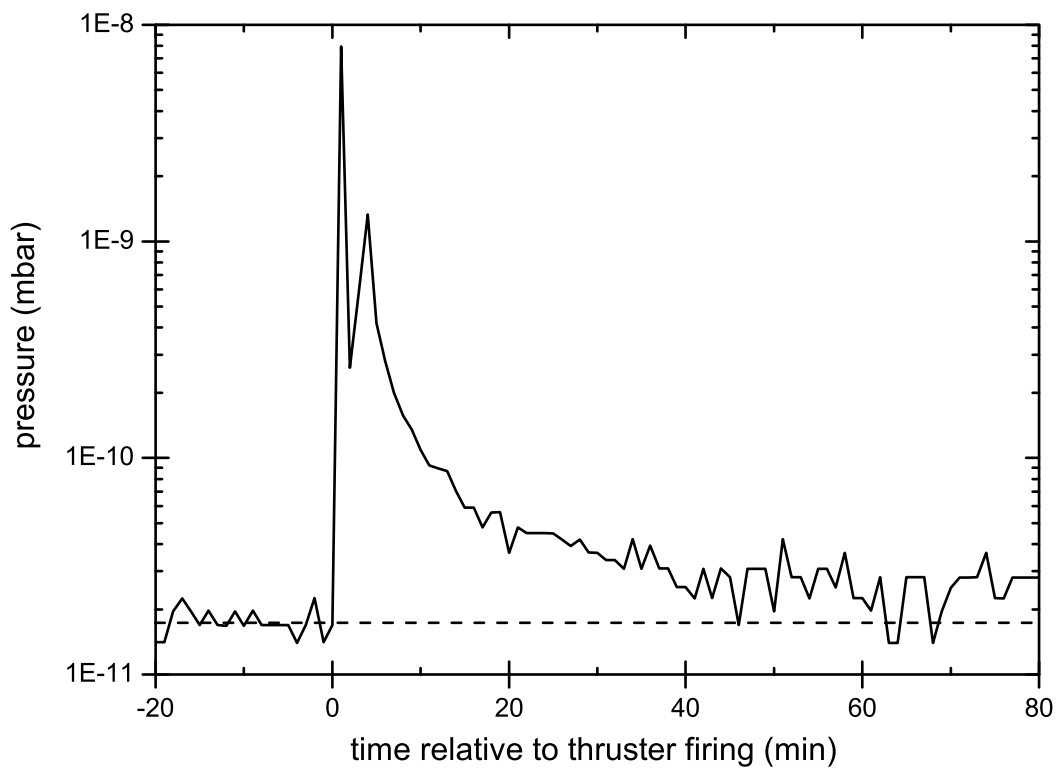

Figure 13. Pressure during and after thruster firing. Multiple thrusters have been activated in this case, which yields pressure peaks of almost three orders of magnitude above the background level. The pressure decreases at the end of the firing within minutes to the $10^{-11}$ mbar range but stays above the background level (dashed line) for more than $1 \mathrm{~h}$.

diffusion leads to a gas cloud around the spacecraft with a density of approximately $10^{5} \mathrm{~cm}^{-3}$ once the desorption process has cleaned the surfaces. Decomposition of the material starts to play a role for long missions after several years in space. Decomposition is mostly attributed to solar UV radiation, which delivers enough activation energy for this process.

[48] While the background may vary somewhat from spacecraft to spacecraft, there is quite a good correspondence between our measurements and those of the MSX and SOHO spacecraft. The partial water pressure is in the low $10^{-11}$ mbar range and appears to be almost generic for all spacecraft; the partial pressure for organic material $(\mathrm{H}-, \mathrm{C}-$, $\mathrm{N}-$, and $\mathrm{O}$-bearing molecules) is one order of magnitude lower.

[49] Backscattered material from spacecraft parts or from self-scattering is deposited preferentially on cold panels of a spacecraft, leading to a large reservoir of volatile material. Unfortunately, the background is not a constant but depends on many parameters such as spacecraft attitude, payload, subsystem operation, etc. Even the composition of the background is not uniform around the spacecraft. Shadowed parts contain mostly water, whereas parts that are mostly in the Sun show a large contribution from decomposition (e.g., lubricants). This may explain that the contamination of sensitive surfaces varies quite a lot from spacecraft to spacecraft and between instruments on the same spacecraft. We can confirm that the contaminant attributed to the lubricant Braycote is an aliphatic compound containing a lot of fluorine as was detected by ACIS/Chandra [Marshall et al., 2004].

[50] This gaseous background clearly interferes with detection by in situ mass spectrometry of weak signals from atmospheres and exospheres of small bodies or bodies with little outgassing. There is no easy solution, as long as the relative spacecraft velocity is small. All scientific spacecraft undergo a stringent contamination control. As long as a spacecraft carries MLIs, honeycomb structures, electronics, payload, insulated wires, etc., it will outgas water and organic material. Once immersed in the atmosphere or exosphere to be investigated, there is almost no way to separate static background from signal. Each spacecraft attitude change may change the background; even closing of a cover may change the local background for a highly sensitive mass spectrometer like DFMS.

[51] There are a few measures that can be taken to mitigate the problem. By modeling outgassing of a spacecraft, there may be the possibility to find a "good" position on the spacecraft for a mass spectrometer, a position where outgassing is minimal. By taking into account the small bulk velocity of the gas to be measured, there is the possibility to apply very small retarding potentials just after the ionization section of the instrument to discriminate background. Because the ionization process also influences the energy of

Table 1. Assumed Production Rates for Calculation of Expected Pressures Around 67P/Churyumov-Gerasimenko ${ }^{\mathrm{a}}$

\begin{tabular}{lccc}
\hline & $\mathrm{H}_{2} \mathrm{O}$ Driven Model & $\mathrm{CO}_{2}$ Driven Model & Perihelion Model \\
\hline \multicolumn{4}{c}{ Heliocentric Distance } \\
& $3 \mathrm{AU}^{\mathrm{b}}$ & $\begin{array}{c}\text { 3AU } \\
\text { Production Rates }\end{array}$ & $1.3 \mathrm{AU}$ \\
$\mathrm{H}_{2} \mathrm{O}\left(\mathrm{s}^{-1}\right)$ & $5.0 \mathrm{E}+25$ & $1.0 \mathrm{E}+26$ & $1.0 \mathrm{E}+28$ \\
$\mathrm{CO}_{2}\left(\mathrm{~s}^{-1}\right)$ & $2.5 \mathrm{E}+24$ & $5.0 \mathrm{E}+24$ & $5.0 \mathrm{E}+26$ \\
$\mathrm{CO}\left(\mathrm{s}^{-1}\right)$ & $5.0 \mathrm{E}+24$ & $1.0 \mathrm{E}+27$ & $1.0 \mathrm{E}+27$ \\
$\mathrm{CH}_{4}\left(\mathrm{~s}^{-1}\right)$ & $2.5 \mathrm{E}+23$ & $5.0 \mathrm{E}+23$ & $5.0 \mathrm{E}+25$ \\
$\mathrm{HCN}\left(\mathrm{s}^{-1}\right)$ & $5.0 \mathrm{E}+22$ & $1.0 \mathrm{E}+23$ & $1.0 \mathrm{E}+25$ \\
\hline
\end{tabular}

${ }^{\mathrm{a}}$ Rates are in rough agreement with other simulations [e.g., Tenishev and Combi, 2008].

${ }^{\mathrm{b}}$ Values represent the heliocentric distance. 


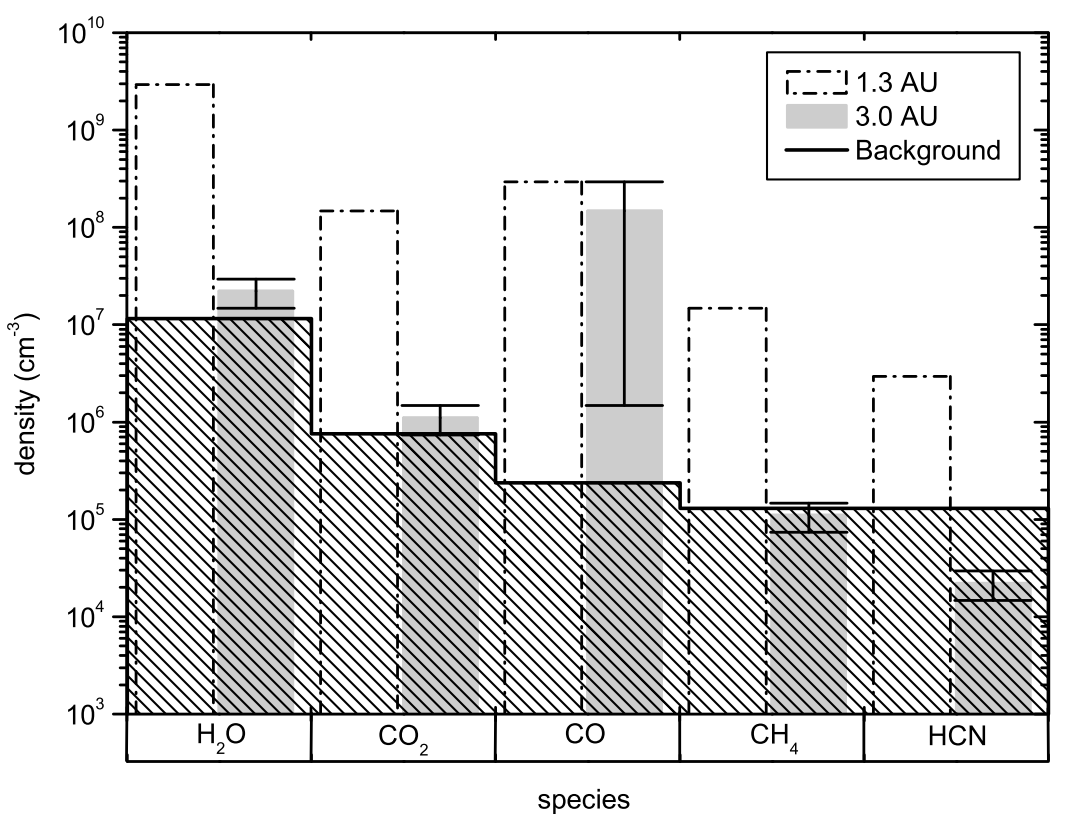

Figure 14. Comparison of ROSINA DFMS background and expected particle densities at Rosetta's final target $67 \mathrm{P} /$ Churyumov-Gerasimenko at $30 \mathrm{~km}$ distance. Gas velocity was assumed to be $300 \mathrm{~m} / \mathrm{s}$. Gray bars are the average of the $\mathrm{CO}_{2}$ - and $\mathrm{H}_{2} \mathrm{O}$-driven early comet activity models ( $3 \mathrm{AU}$ heliocentric distance). Errors depict the variations between the two models. The background may be somewhat lower for the $3 \mathrm{AU}$ case because of the larger heliocentric distance.

the ions, this may not be feasible for bulk velocities below $1 \mathrm{~km} / \mathrm{s}$ or for the light masses $\left(\mathrm{H}, \mathrm{H}_{2}, \mathrm{He}, \mathrm{C}\right.$, etc.). It could also be foreseen to use a very small shutter mechanism a short distance in front of the mass spectrometer entrance. Background may be determined by measuring in a quasi phase locked loop with and without shutter. All of these measures could reduce the background problem somewhat, but not by many orders of magnitude. Thus, because of the presence of the spacecraft, enhanced sensitivities of mass spectrometers will not necessarily lead to lower detection limits for atmospheres and exospheres.

[52] For the ROSINA instruments, these findings are crucial for future operations during the second half of the mission: in July 2010, Rosetta will flyby the asteroid (21) Lutetia, where ROSINA will be used to investigate whether this body exhibits some low-level activity [Schläppi et al., 2008]. It is planed to perform a flyby rehearsal to identify possible spacecraft outbursts at given attitudes. Comparison of the rehearsal and the flyby data should allow us to derive a sensitive upper limit of Lutetia's outgassing rate.

[53] During early comet operations at a heliocentric distance $>3 \mathrm{AU}$ in 2014, ROSINA data analysis will have to be done with extreme caution to distinguish "false comet signals" arising from spacecraft outgassing from the real cometary signature. To quantify these interferences, an isotropic radial outflow model for the comet without sources or sinks was calculated for three different cases (Table 1).

[54] With these parameters, it is expected that ROSINA will be able to identify major cometary species $\left(\mathrm{H}_{2} \mathrm{O}, \mathrm{CO}\right.$ or $\mathrm{CO}_{2}$ ) at large heliocentric distances $>3 \mathrm{AU}$. However, as Figure 14 clearly demonstrates, to detect minor species, such as $\mathrm{HCN}$ or $\mathrm{CH}_{4}$, specific measurement methods will be necessary at $3 \mathrm{AU}$. At $1.3 \mathrm{AU}$, close to perihelion, the interference will be negligible.
[55] Acknowledgments. We thank the following institutions and agencies that supported this work. Work at the University of Bern was funded by the State of Bern, the Swiss National Science Foundation, and the European Space Agency PRODEX Program. Work at BIRA-IASB was supported by the Belgian Science Policy Office via PRODEX/ROSINA PEA 90020. Research at the Lockheed Martin Advanced Technology Center and work at the University of Michigan were supported under JPL subcontract 1266313 under NASA prime contract NMO710889. The work on ROSINA in France was supported by CNES through grants CNES/ROSETTA/98-70 to 04-70. We also thank the Flight Operations group at ESOC, which provided us with the opportunities to study the outgassing behavior of Rosetta during special tests.

[56] Philippa Browning thanks the reviewers for their assistance in evaluating this paper.

\section{References}

Acton, C. H. (1996), Ancillary data services of NASA's Navigation and Ancillary Information Facility, Planet. Space Sci., 44(1), 65-70, doi:10.1016/0032-0633(95)00107-7.

Balsiger, H., et al. (2007), ROSINA - Rosetta Orbiter Spectrometer for Ion and Neutral Analysis, Space Sci. Rev., 128, 745-801, doi:10.1007/ s11214-006-8335-3.

Bhaskaran, S., N. Mastrodemos, J. E. Riedel, and S. P. Synnott (2004), Optical navigation for the STARDUST Wild 2 Encounter, paper presented at 18th International Symposium on Space Flight Dynamics (ESA SP-548), Ger. Space Oper. Cent. of DLR and Eur. Space Oper. Cent. of ESA, Munich, Germany.

Bibring, J.-P., et al. (2007), The Rosetta Lander ("PHILAE") investigations, Space Sci. Rev., 128, 205-220, doi:10.1007/s11214-006-9138-2.

Brinza, D., J. Wang, J. Polk, and M. Henry (2000), Deep Space One investigations of ion propulsion contamination: Overview and initial results, paper 2000-0465, paper presented at 38th Aerospace Sciences Meeting and Exhibit, Am. Inst. of Aeronaut. and Astronaut., Reno, Nev.

Campbell, W. A., and J. J. Scialdone (1993), Outgassing data for selecting spacecraft materials, NASA Ref. Publ. 1124, Rev. 3, NASA, Greenbelt, Md.

Chen, J. R., G. Y. Hsiung, Y. J. Hsu, S. H. Chang, C. H. Chen, W. S. Lee, J. Y. Ku, C. K. Chan, L. W. Joung, and W. T. Chou (2001), Water adsorption-desorption on aluminum surface, Appl. Surf. Sci., 169-170, 679-684, doi:10.1016/S0169-4332(00)00812-6. 
Colangeli, L., et al. (2007), The Grain Impact Analyser and Dust Accumulator (GIADA) experiment for the Rosetta mission: Design, performances and first results, Space Sci. Rev., 128, 803-821, doi:10.1007/s11214-0069038-5.

Coradini, A., et al. (2007), Virtis: An imaging spectrometer for the Rosetta mission, Space Sci. Rev., 128, 529-559, doi:10.1007/s11214-006-9127-5.

de Bonn, O., A. Hammerl, T. M. Klapötke, P. Mayer, H. Piotrowski, and H. Zewen (2001), Plume deposits from bipropellant rocket engines: Methylhydrazinium nitrate and N,N-dimethylhydrazinium nitrate, $Z$. Anorg. Allg. Chem., 627, 2011-2015, doi:10.1002/1521-3749(200108) 627:8<2011::AID-ZAAC2011>3.0.CO;2-G.

Defise, J.-M., F. Clette, J. D. Moses, and J.-F. E. Hochedez (1997), In-orbit diagnostics of EIT EUV CCD radiation-induced aging, Proc. SPIE, 3114, 598-607.

Glassmeier, K.-H., H. Boehnhardt, D. Koschny, E. Kührt, and I. Richter (2007), The Rosetta mission: Flying towards the origin of the solar system, Space Sci. Rev., 128, 1-21, doi:10.1007/s11214-006-9140-8.

Graf, S., K. Altwegg, H. Balsiger, P. Bochsler, B. Fiethe, and E. Montagnon (2008), Thruster plumes: Sources for high pressure and contamination at the payload location, J. Spacecr. Rockets, 45(1), 57-64, doi:10.2514/ 1.30600

Green, B. D. (2001), Satellite Contamination and Materials Outgassing Knowledgebase - An Interactive Database Reference, NASA/CR-2001210909, prepared for NASA's Space Environ. and Effects (SEE) Program Tech. Monit., edited by Dewitt Burns, 131 pp., George C. Marshall Space Flight Cent., Ala.

Haemmerle, V. R., and J. H. Gerhard (2006), Cassini camera contamination anomaly: Experiences and lessons learned, Jet Propul. Lab., NASA, Pasadena, Calif. (Available at http://hdl.handle.net/2014/40797).

Hoffman, J. H., R. R. Hodges Jr., and D. E. Evans (1972), Lunar orbital mass spectrometer experiment, proceedings of the 3rd Lunar Science Conference, Geochim. Cosmochim. Acta, 3(suppl. 3), 2205-2216.

Marshall, H. L., A. Tennant, C. E. Grant, A. P. Hitchcock, S. O'Dell, and P. P. Plucinsky (2004), Composition of the Chandra ACIS contaminant, Proc. SPIE, 5165, 497-508.

McFadden, J. P., et al. (2007), Flight instrument calibration and performance verification, in Calibration of Particle Instruments in Space Physics, ISSI Sci. Rep. SR-07, pp. 277-385, Int. Space Sci. Inst., Bern, Switzerland.

McMullin, D. R., D. L. Judge, M. Hilchenbach, F. Ipavich, P. Bochsler, P. Wurz, A. Bürgi, W. T. Thompson, and J. S. Newmark (2002), In-flight comparisons of solar EUV irradiance measurements provided by the CELIAS/SEM on SOHO, in Radiometric Calibration of SOHO, ISSI Sci. Rep. SR-02, pp. 135-144, Int. Space Sci. Inst., Bern, Switzerland.

Pereira, C. (2001), Mechanisms of the Rosetta high gain antenna, in Proceedings of the Ninth European Space Mechanisms and Tribology Symposium, ESA SP-480, edited by R. A. Harris, pp. 83-90, Noordwijk, Netherlands.

Plucinsky, P. P., et al. (2003), The flight spectral response of the ACIS instrument, Proc. SPIE, 4851, 89-100, doi:10.1117/12.461473.

Robertson, S. J. (1976), Spacecraft self-contamination due to back-scattering of outgas products, Interim Rep. LMSC-HREC TR D496676, Lockheed Missions and Space Co., Palo Alto, Calif.

Schläppi, B., K. Altwegg, and P. Wurz (2008), Asteroid exosphere: A simulation for the ROSETTA flyby targets (2867) Steins and (21) Lutetia Icarus, 195(2), 674-685, doi:10.1016/j.icarus.2007.12.021.
Schulz, R., J. A. Stüwe, and H. Boehnhardt (2004), Rosetta target 67P/ Churyumov-Gerasimenko - Postperihelion gas and dust production rates, Astron. Astrophys., 422, L19-L21, doi:10.1051/0004-6361:20040190.

Scialdone, J. J., A. E. Hedin, and C. J. Rice (1978), Comparison of Satellite Self-Contamination Experiments and Scattering Return Flux Calculations, J. Geophys. Res., 83 (A1), 195-198.

Tenishev, V., and M. Combi (2008), A global kinetic model for the cometary coma: The evolution of the coma of the Rosetta target comet Churyumov-Gerasimenko throughout the mission, Astrophys. J., 685 , 659-677, doi:10.1086/590376.

Thomas, P. D., M. C. Fong, and K. L. Neier (1998), Return flux of neutral and charged particles in geosynchronous orbit, Proc. SPIE, 3427, 290, doi:10.1117/12.328501.

Tribble, A. C. (2000), Fundamentals of Contamination Control, SPIE Press, Bellingham, Wash., doi:10.1117/3.387881.

Uy, O. M., B. D. Green, B. E. Wood, G. E. Galica, M. T. Boies, J. C. Lesho, R. Cain, and D. F. Hall (2003), The gaseous and particle environment observed above the MSX spacecraft after seven years on orbit, paper presented at Ninth International Symposium on Materials in a Space Environment, ESA SP-540, Eur. Space Agency, Noordwijk, Netherlands.

Waite, J. H., Jr., et al. (2004), The Cassini Ion and Neutral Mass Spectrometer (INMS) investigation, Space Sci. Rev., 114(1-4), 113-231, doi:10.1007/ s11214-004-1408-2.

Wood, B. E., D. F. Hall, J. C. Lesho, O. M. Uy, J. S. Dyer, and W. T. Bertrand (1997), MSX satellite flight measurements of contaminant deposition on a CQCM and on TQCMs, Paper 97-0841, paper presented at 35th Aerospace Sciences Meeting and Exhibit, Am. Inst. of Aeronaut. and Astronaut., Reno, Nev.

Wright, I. P., et al. (2007), PTOLEMY - An instrument to measure stable isotopic ratios of key volatiles on a cometary nucleus, Space Sci. Rev., 128, 363-381, doi:10.1007/s11214-006-9001-5.

K. Altwegg, H. Balsiger, M. Hässig, A. Jäckel, B. Schläppi, and P. Wurz, Physikalisches Institut, University of Bern, Sidlerstrasse 5, CH-3012 Bern, Switzerland. (schlaeppi@space.unibe.ch)

J. J. Berthelier, LATMOS, 4 Av. de Neptune, F-94100 Saint-Maur, France.

J. De Keyser, Space Physics Division, BIRA-IASB, Ringlaan 3, B-1180 Brussels, Belgium.

B. Fiethe, Institute of Computer and Network Engineering, TU Braunschweig, Hans-Sommer-Straße 66, D-38106 Braunschweig, Germany.

S. A. Fuselier, Space Physics Department, Lockheed Martin Advanced Technology Center, Department ADCS, Bldg. 255, 3251 Hanover St., Palo Alto, CA 94304, USA.

U. Mall, Max-Planck-Institut für Sonnensystemforschung, MaxPlanckstrasse 2, D-37191 Katlenburg-Lindau, Germany.

H. Rème, UPS, CESR, University of Toulouse, 9 Av. du Colonel Roche, F-31028 Toulouse CEDEX 4, France.

M. Rubin, AOSS, University of Michigan, Space Research Building, 2455 Hayward St., Ann Arbor, MI 48109, USA. 